\title{
STRUCTURE IN THE GAS AND MAGNETIC FIELD IN S106
}

\author{
RICHARD M. CRUTCHER \\ Astronomy Department \\ University of Illinois \\ Urbana, IL 61801 \\ U.S.A.
}

ABSTRACT. BIMA molecular-line observations show evidence for an expanding molecular ring around IRS 4, a newly formed massive star at the center of the bipolar nebula S106. VLA observations of the Zeeman effect in the $\mathrm{OH} 1665 \mathrm{MHz}$ line show that the magnetic field strength is about $1 \mathrm{mG}$ and that it reverses direction from one lobe of the bipolar nebula to the other.

\section{Introduction}

S106 is a biconical H II region and a site of recent star formation; it consists of a dark lane which separates two lobes of $\mathrm{H}$ II flowing away from a newly formed massive star, IRS 4 . The entire complex is embedded in the placental molecular cloud from which it formed. We have mapped the small scale structure of the gas and of the magnetic field in this region. The Berkeley-IllinoisMaryland millimeter-wave Array was used to map the 3-mm emission lines of $\mathrm{HCO}^{+}, \mathrm{H}^{13} \mathrm{CN}$, and CS. The VLA was used to map the Stokes parameter I and V spectra of the $\mathrm{OH} 1665 \mathrm{MHz}$ absorption line.

\section{BIMA Observations}

The BIMA observations of the $\lambda=3 \mathrm{~mm}$ lines of $\mathrm{HCO}^{+}, \mathrm{H}^{13} \mathrm{CN}$, and $\mathrm{CS}$ produced maps with an angular resolution of about 8 arcseconds and a velocity resolution of $1 \mathrm{~km} \mathrm{~s}^{-1}$. All maps were centered on IRS $4\left[\alpha(1950)=20^{\mathrm{h}} 25^{\mathrm{m}} 33.8^{\mathrm{s}}, \delta(1950)=37^{\circ} 12^{\prime} 50^{\prime \prime}\right]$ and cover the full-width-athalf-power diameter of the 6-m BIMA antennas, about 2 arcminutes. Figure 1 shows the $\mathrm{HCO}^{+}$ data summed over the velocity extent of the line. The brightest peak in the $\mathrm{HCO}^{+}$emission lies at the northwest corner of the southern ionized lobe. Other peaks occur near each of the other three corners of the ionized lobes; on the eastern side of the H II, there is a north-south ridge of $\mathrm{HCO}^{+}$emission. IRS 4 lies in a relative minimum of emission, with emission extending east-west just to the north and to the south of IRS 4. To investigate the kinematics of the dark lane region between the ionized lobes, we made a position-velocity cut (figure 2) through IRS 4 parallel to the dark lane separating the two ionized lobes. The dark lane extends \pm 20 " from IRS 4. Outside this region, there is little or no velocity gradient; the line width is about $2 \mathrm{~km} \mathrm{~s}^{-1}$. Within the dark lane region, there is an incomplete velocity ellipsoid, with the positive velocity gas to the west

61

E. Falgarone et al. (eds.), Fragmentation of Molecular Clouds and Star Formation, 61-64.

(c) 1991 IAU. Printed in the Netherlands. 


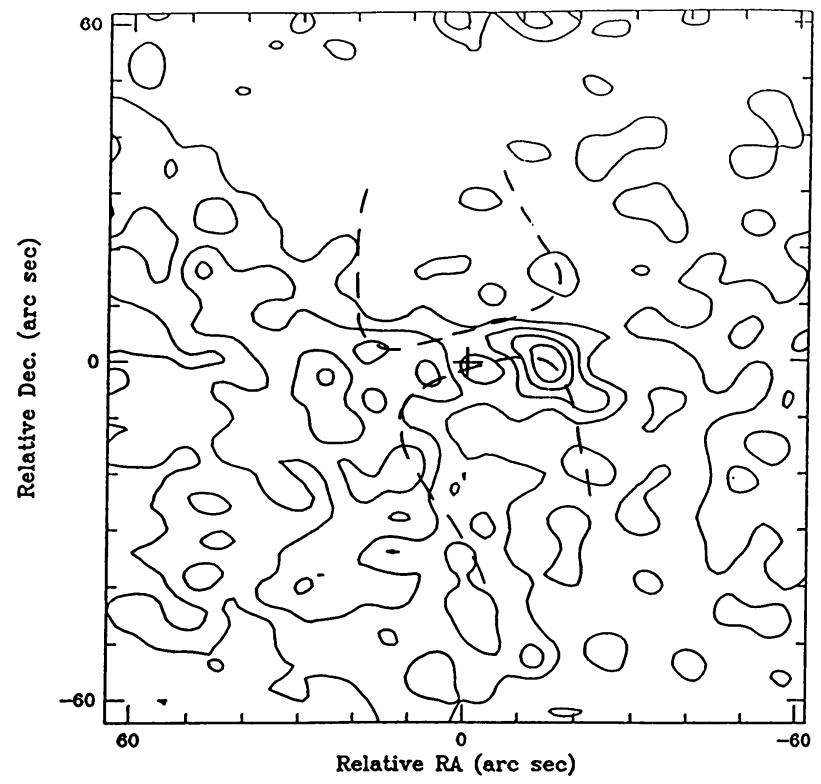

Figure 1. Contours show $\mathrm{HCO}^{+}$emission summed over velocity. Coordinates are with respect to IRS 4, which is shown as a cross at the center. Dashed contours mark the outer boundary of the $\mathrm{H}$ II region as delineated by VLA continuum observations.

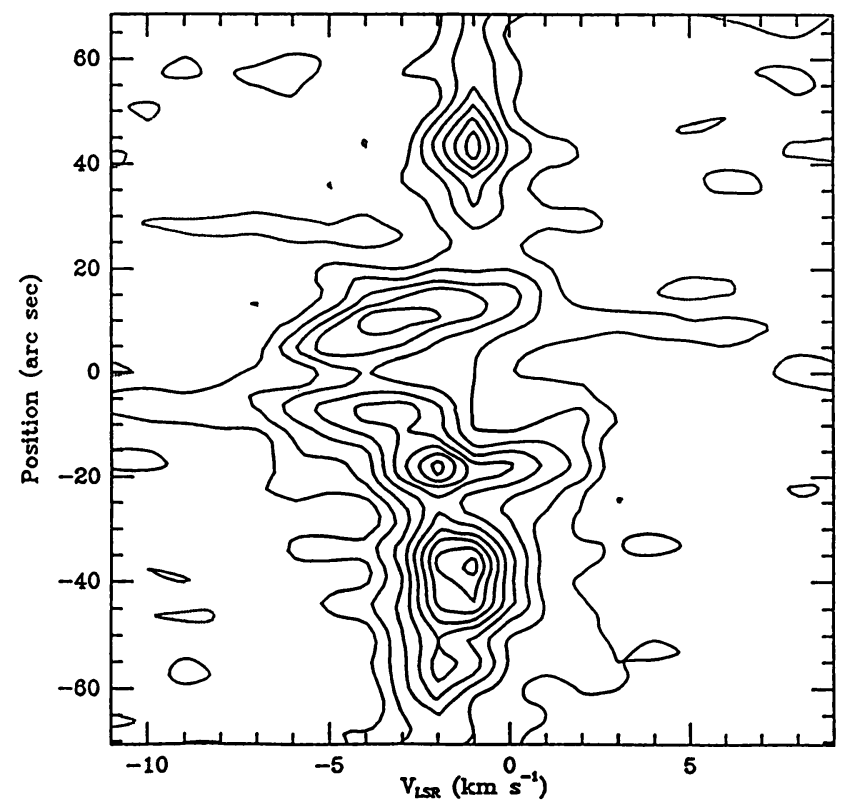

Figure 2. Position-velocity cut through IRS 4 from east to west at position angle $115^{\circ}$. 
being missing. Also, there are higher velocity clumps of gas, seen at $\left(+10^{\prime \prime},>3 \mathrm{~km} \mathrm{~s}^{-1}\right)$ and at $\left(-5^{\prime \prime},<-6 \mathrm{~km} \mathrm{~s}^{-1}\right)$ in figure 2; additional such clumps are seen at other positions north and south of the dark lane.

Our interpretation of these data is that there is a clumpy molecular ring around IRS 4 . The radius of the ring is about $2 \times 10^{17} \mathrm{~cm}$ and its mass is $\leq 10 M_{\odot}$. The ring is not rotating, but it is expanding with a velocity of about $2 \mathrm{~km} \mathrm{~s}^{-1}$. This ring may be the residual of a disk of cold, dense material which developed during the formation of IRS 4 and which initially directed the ionized outflow from IRS 4 into a bipolar morphology. The star has begun to dissipate its surrounding placental material, so that the disk is now almost gone. The clumps of high velocity material which are observed may have been ablated from the ring and entrained in the ionized bipolar outflow.

The molecular cloud surrounding the $\mathbf{S 1 0 6}$ region is also very clumpy, although unlike the dark lane region there is very little velocity structure. The maps suggest that gas traced by CS and $\mathrm{H}^{13} \mathrm{CN}$ emission is relatively quiescent and located in a cocoon-like structure surrounding the ionized lobes. On the other hand, $\mathrm{HCO}^{+}$emission peaks closer to the ionized regions than $\mathrm{CS}$ and $\mathrm{HCN}$. The $\mathrm{HCO}^{+}$is enhanced just outside continuum peaks where the ionized outflow appears to encounter the surrounding molecular cloud and to be deflected northward and southward into the lobes. The $\mathrm{HCO}^{+}$enhancement may be a result of shock chemistry.

\section{VLA Observations}

The VLA was used to map Stokes parameter I and V spectra for the $1665 \mathrm{MHz}$ line of $\mathrm{OH}$ in absorption toward the S106 continuum. A continuum map was produced from spectrometer channels with no line signal. Brief observations were made of the $1720 \mathrm{MHz}$ maser. The angular resolution was about $10^{\prime \prime}$ and the velocity resolution $0.5 \mathrm{~km} \mathrm{~s}^{-1}$. Maps of the optical depth of the $\mathrm{OH}$ line were made from the continuum and I data. The line-of-sight component of the magnetic field strength and its direction were inferred from the $\mathrm{V}$ spectra.

The $\mathrm{OH}$ absorption is strongest at about $0 \mathrm{~km} \mathrm{~s}^{-1}$, with a gradually decreasing strength out to about $-10 \mathrm{~km} \mathrm{~s}^{-1}$. The ring is seen in absorption in $\mathrm{OH}$ with a velocity which shows that the ring is expanding. $\mathrm{OH}$ absorption against the ionized lobes is very clumpy; these clumps are responsible for the long negative velocity tail to the $\mathrm{OH}$ line profile. This $\mathrm{OH}$ appears to be confined to the interface between the ionized lobes and the surrounding molecular gas, and is interpreted as material being entrained in the bipolar outflow.

The magnetic field in S106 measured by single-antenna Zeeman experiments is about 0.1 milligauss. The VLA results are shown in figure 2; they show that the field reverses direction from one lobe to the other, with a typical magnitude of about 1 milligauss. This result is consistent with the magnetic field having an hourglass morphology, with the maser behind the $\mathrm{H}$ II of the northern lobe and the $\mathrm{OH}$ absorption tracing the field at the front interface between the H II and the surrounding molecular cloud. This morphology is that expected for a centrally condensed cloud which has contracted with flux freezing. The magnetic field strength is clearly large enough to be important in the dynamics of the region. Since the molecular ring is not observed to be rotating, magnetic fields could be playing a dominant role in its support. 


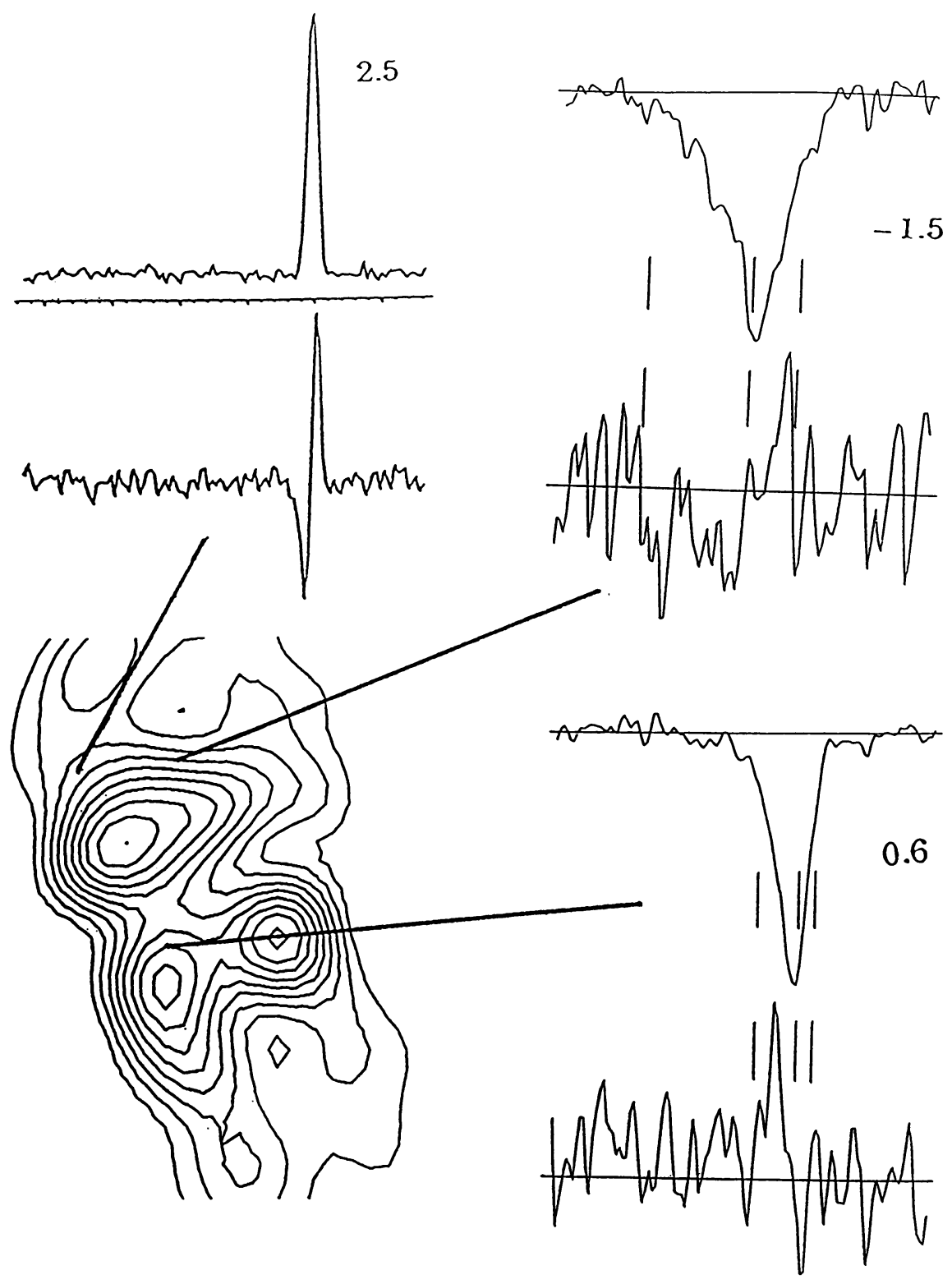

Figure 3. The contours at bottom left show our $18 \mathrm{~cm}$ VLA continuum map. Stokes parameter I (upper) and $\mathrm{V}$ (lower) profiles are shown for the $1720 \mathrm{MHz}$ maser (upper left) and for two positions for the $1665 \mathrm{MHz}$ absorption (right). Lines show the positions on the continuum where each of the profiles was obtained. Magnetic fields are $+2.5 \mathrm{mG}$ for the maser, $-1.5 \mathrm{mG}$ for the northern lobe in $\mathrm{OH}$ absorption, and $+0.6 \mathrm{mG}$ for the southern lobe in absorption. 